\title{
Certifying an Irreducible 1024-Dimensional Photonic State Using Refined Dimension Witnesses
}

\author{
Edgar A. Aguilar, ${ }^{1}$ Máté Farkas, ${ }^{1}$ Daniel Martínez, ${ }^{2,3}$ Matías Alvarado, ${ }^{2,3}$ Jaime Cariñe, ${ }^{2,3}$ Guilherme B. Xavier, ${ }^{3,45}$ Johanna \\ F. Barra, ${ }^{2,3}$ Gustavo Cañas, ${ }^{6}$ Marcin Pawłowski, ${ }^{1}$ and Gustavo Lima ${ }^{2,3}$ \\ ${ }^{1}$ Institute of Theoretical Physics and Astrophysics, National Quantum Information Centre, \\ Faculty of Mathematics, Physics and Informatics, University of Gdansk, 80-952 Gdansk, Poland \\ ${ }^{2}$ Departamento de Física, Universidad de Concepción, 160-C Concepción, Chile \\ ${ }^{3}$ Millennium Institute for Research in Optics, Universidad de Concepción, 160-C Concepción, Chile \\ ${ }^{4}$ Departamento de Ingeniería Eléctrica, Universidad de Concepción, 160-C Concepción, Chile \\ ${ }^{5}$ Institutionen för Systemteknik, Linköpings Universitet, 58183 Linköping, Sweden \\ ${ }^{6}$ Departamento de Física, Universidad del Bio-Bio, Avenida Collao 1202, Concepción, Chile
}

(Received 22 September 2017; revised manuscript received 23 March 2018; published 6 June 2018)

\begin{abstract}
We report on a new class of dimension witnesses, based on quantum random access codes, which are a function of the recorded statistics and that have different bounds for all possible decompositions of a highdimensional physical system. Thus, it certifies the dimension of the system and has the new distinct feature of identifying whether the high-dimensional system is decomposable in terms of lower dimensional subsystems. To demonstrate the practicability of this technique, we used it to experimentally certify the generation of an irreducible 1024-dimensional photonic quantum state. Therefore, certifying that the state is not multipartite or encoded using noncoupled different degrees of freedom of a single photon. Our protocol should find applications in a broad class of modern quantum information experiments addressing the generation of high-dimensional quantum systems, where quantum tomography may become intractable.
\end{abstract}

DOI: $10.1103 /$ PhysRevLett.120.230503

Introduction.-The dimension $d$ of physical systems is a fundamental property of any model, and its operational definition arguably reflects the evolution of physics itself. In quantum mechanics, it can be seen as a key resource for information processing since higher dimensional systems provide advantages in several protocols of quantum computation [1] and quantum communications [2]. In the field of quantum foundations, a recent proposal suggests that, in order to understand and create macroscopic quantum states, it will be necessary to take advantage of high-dimensional systems [3]. Therefore, it is natural to understand why there is a growing endeavor to coherently control quantum systems of large dimensions [4-16]. Nonetheless, such new technological advances require the simultaneous development of practical methods to certify that the sources are truly producing the required quantum states. In principle, one can rely on the process of quantum tomography [17-23], but this approach quickly becomes intractable in higher dimensions as at least $d^{2}$ measurements are required [24].

To address this problem, the concept of dimension witness (DW) was introduced. The original idea was based on the violation of a particular Bell inequality [25] but was then extended to the more practical prepare-and-measure scenario [26]. In general, DWs are defined as linear functions of a few measurement outcome probabilities and have classical and quantum bounds defined for each considered dimension $[4,25-30]$. Thus, they allow for the device-independent certification of the minimum dimension required to describe a given physical system and can also infer whether it is properly described by a coherent superposition of logical states. Nevertheless, these tests do not provide information about the composition of the system, which is crucial for high-dimensional quantum information processing. This point has been recently investigated by W. Cong et al. [31], where they introduced the concept of an irreducible dimension witness (IDW) to certify the presence of an irreducible four dimensional system. Specifically, their IDW distinguishes whether if the observed data are created by one pair of entangled ququarts, or two pairs of entangled qubits measured under sequential adaptive operations and classical communication.

Here, we introduce a new class of DWs, namely gamut DWs, which certifies the dimension of the system and has the new distinct feature of identifying whether any highdimensional quantum system is irreducible. It is based on quantum random access codes (QRACs), which is a communication task defined in a prepare-and-measure scenario [32]. To demonstrate the practicability of our new technique, we experimentally certify the generation of an irreducible 1024-dimensional photonic quantum system encoded onto the transverse momentum of single photons transmitted over programmable diffractive optical devices [5,21-23,33-35]. To our knowledge, our work represents an increase of about 2 orders of magnitude to any reported 
experiment using path qudits. From the recorded data, one observes a violation of the bounds associated with all possible decompositions of a 1024-dimensional quantum system, thus, certifying that the generated state is not encoded using noncoupled different degrees of freedom of a photon, e.g., polarization and momentum. Nonetheless, our method is broadly relevant and should also find applications in multipartite photonic scenarios and new platforms for the fast-growing field of experimental highdimensional quantum information processing.

Gamut dimension witness. - As stated earlier, the protocol we use in our main theorem is based on QRACs. Thus, first, we give a brief description (see, e.g., [32] for more details) of this task (see Fig. 1): one of the parties, Alice, receives two input dits: $x_{1}$ and $x_{2} \in\{1, \ldots, d\}$. She is then allowed to send one $d$-dimensional (quantum) state, $\rho_{x_{1} x_{2}}$ to Bob, depending on her input. Bob is then given a bit $y \in\{1,2\}$ and his task is to guess $x_{y}$. He does so by performing a quantum measurement $M^{y}$ and a classical post-processing function $\mathcal{D}^{y}$. As a result, he outputs $b \in\{1, \ldots, d\}$.

For a single round of the protocol, the success probability is $\mathbb{P}\left(b=x_{y} \mid x_{1}, x_{2}, y\right)$. As a figure or merit over many rounds with uniformly random inputs, we employ the average success probability (ASP): $\bar{p}=$ $\left(1 / 2 d^{2}\right) \sum_{x_{1}, x_{2}, y} \mathbb{P}\left(b=x_{y} \mid x_{1}, x_{2}, y\right)$. Thus, we are looking for the maximal value of $\bar{p}$, optimizing over all possible encoding and decoding strategies. It was proven [36] that, for classical strategies (i.e., classical states and decoding functions), the optimal ASP is $\bar{p}_{C_{d}}=\frac{1}{2}(1+1 / d)$. In the quantum case, the optimal strategy is reached by using mutually unbiased bases (MUBs) for encoding and decoding $[37,38]$, and the ASP is $\bar{p}_{Q_{d}}=\frac{1}{2}(1+1 / \sqrt{d})$.

Now, we estimate the optimal ASPs for composite systems, for all possible product structures, defined as follows.

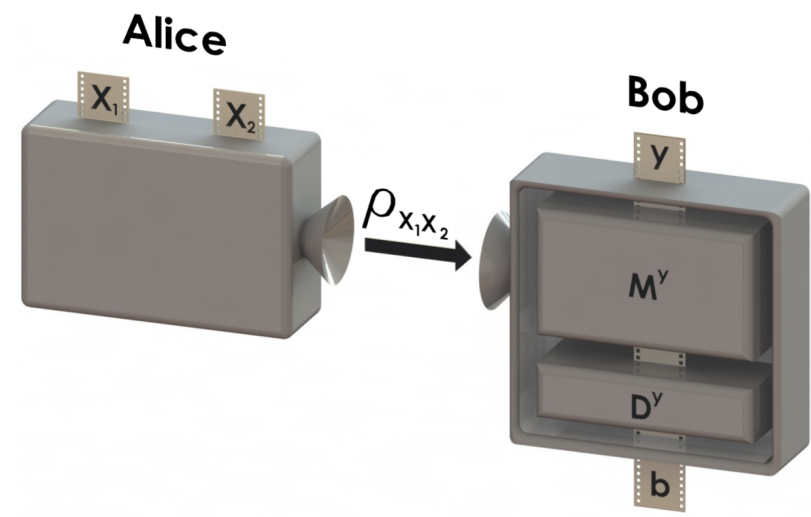

FIG. 1. Our $d$-dimensional QRACs scenario. Alice receives the input dits $x_{1}$ and $x_{2} \in\{1, \ldots, d\}$, and prepares the state $\rho_{x_{1} x_{2}}$ which is sent to Bob. He receives the input $y \in\{1,2\}$, which defines the quantum measurement $M^{y}$ and the classical postprocessing function $\mathcal{D}^{y}$ to be applied to $\rho_{x_{1} x_{2}}$. As a result, Bob outputs $b$.
Definition 1.-For a fixed $d$, we define a product structure by the set $\left\{r,\left\{d_{k}\right\},\left\{\alpha_{k}\right\}\right\}$. For a composite system, $d=\prod_{k=1}^{r} d_{k}$, where $d_{k}$ is the dimension of each subsystem and $r$ is the number of subsystems. The state of the composite system can be written as $\rho=\rho_{\alpha_{1}}^{1} \otimes \rho_{\alpha_{2}}^{2} \otimes$ $\cdots \otimes \rho_{\alpha_{r}}^{r}$. Here, $\alpha_{k}=\mathrm{c}$ and $\alpha_{k}=\mathrm{q}$ are used to denote the "classical" and "quantum" nature of the subsystem, respectively. Then, $\rho_{c}^{k} \in \Delta_{d_{k}-1}$ is a classical state, and $\rho_{q}^{k} \in$ $\mathcal{S}\left(\mathbb{C}^{d_{k}}\right)$ is a quantum state.

Consider a set of measurement and state preparation settings and fix the total dimension of the physical system in question. We call a linear function on the measurement outcome probabilities a gamut dimension witness (GDW) if its extremal values for all possible product structures are different. For example, in $d=4$, a GDW has different extremal values for a ququart, two qubits, one qubit and a bit, and a quart. The main theoretical result of this work is to demonstrate that $d$-dimensional QRACs can be used as GDWs for $d$-dimensional physical systems. To highlight this, we set it as a theorem.

Theorem 1.- $d$-dimensional QRACs serve as gamut dimension witnesses using the ASP function.

The proof of this theorem and all related lemmas can be found in the Supplemental Material [39]. Let us now sketch the main tools for proving the theorem. They help to understanding the problem, and can be independently used. Note that the following lemmas apply in more general QRAC scenarios as well [39].

We assume that Bob's measurements have the same product structure as the state generated by Alice. That is, we exclude that Bob's state certification would use entangling measurements. The motivation here is to rule out sequential uses of lower dimensional systems as a way to simulate higher dimensional statistics, e.g., to discriminate between $n$ sequential uses of a $d$-dimensional system, and a $d^{n}$-dimensional system. A physical motivation for this assumption is to think that, if Alice cannot couple a particular set of degrees of freedom (e.g., polarization and momentum), then neither can Bob because he has access to the same equipment as Alice does [43].

Therefore, the most general strategy for decoding the $d$-dimensional system $\rho=\rho^{1} \otimes \rho^{2} \otimes \cdots \otimes \rho^{r}$ is as follows: Bob performs sequential adaptive measures on the subsystems in the sense of [31]. He starts by measuring subsystem $\rho^{1}$ to obtain the outcome $b^{1}$. Then, his choice of the measurement to be performed in $\rho^{2}$ may depend on $b^{1}$. Successively, each measurement on $\rho^{k}$ can depend on all the measurement outcomes obtained previously. After performing all measurements, Bob feeds the obtained outcomes to a classical post-processing function and outputs his final guess on $x_{y}$, which is $b=\mathcal{D}^{y}\left(b^{1} b^{2}, \ldots, b^{r}\right)$.

The bounds of the GDW in this general scenario are extremely hard to obtain. The following results help, making the analysis easier. First, it is argued in [32] that, 
in an optimal strategy, it is enough to use encoded pure states. Similarly, it has been shown that rank 1 projective measurements (explicitly: mutually unbiased bases) optimize two-input QRACs [38]. Thus, in the following, we only deal with pure states for both Alice and Bob. Additionally, we can eliminate classical post-processing functions.

Lemma 1.-In QRACs, for optimality of the ASP, there is no need for classical post-processing functions.

Last, we note that:

Lemma 2.-In QRACs, for optimality of the ASP, there is no need for sequential adaptive measurements.

Observe that the above lemmas together imply that the highest ASP for a composite system can be achieved with a strategy that consists of $r$ QRACs in parallel, one on each subsystem $\rho^{k}$, independently. In this case, if we write Alice's inputs as dit strings $x_{y}=x_{y}^{1} x_{y}^{2}, \ldots, x_{y}^{r}$, the success probability for each round is $\mathbb{P}\left(b=x_{y} \mid x_{1}, x_{2}, y\right)=\prod_{k=1}^{r} \mathbb{P}\left(b^{k}=\right.$ $\left.x_{y}^{k} \mid x_{1}^{k}, x_{2}^{k}, y\right)$. The optimal $\bar{p}$ is not necessarily given by the independent optimal strategies on the individual subspaces. Therefore, in order to optimize it we introduce the trade-off function $\mathcal{M}_{d}(z)$ (see the Supplemental Material [39]), which provides the optimal probability of guessing dit $x_{2}$ given a fixed probability of guessing dit $x_{1}$. Let $z=$ $\mathbb{P}\left(\right.$ Bob correctly guesses $\left.x_{1}\right)$. Then, $\mathcal{M}_{d}(z)$ in dimension $d$ is defined by $\mathcal{M}_{d}(z)=\max \left\{\mathbb{P}\left(\right.\right.$ Bob correctly guesses $\left.\left.x_{2}\right) \mid z\right\}$, where the maximization is limited to all encoding-decoding strategies respecting the condition of guessing $x_{1}$ with probability $z$. Thus, in a general case,

$\bar{p}_{Q_{d_{1}}, \ldots, C_{d_{r}}}=\max _{z^{1}, \ldots, z^{r}} \frac{1}{2}\left[z^{1} \cdots z^{r}+\mathcal{M}_{d_{1}}^{q}\left(z^{1}\right) \cdots \mathcal{M}_{d_{r}}^{c}\left(z^{r}\right)\right]$,

where we denote $d$-dimensional quantum and classical states by $Q_{d}$ and $C_{d}$, respectively. $\mathcal{M}_{d}^{q}$ and $\mathcal{M}_{d}^{c}$ are the corresponding quantum, and classical trade-off functions [39]. Therefore, $\bar{p}$ is a function of $r$ real variables, and its maximum can be found using standard heuristic numerical search algorithms [44]. We present the ASP optimal values for some relevant cases of a $d=1024$ dimensional system in Table I. The full list of cases is found in the Supplemental Material [39]. Note that the gaps between the different ASP values are large enough to be experimentally observed, as we demonstrate next.

Experiment.-To demonstrate the practicability of our technique, we generate a 1024-dimensional photonic state, encoded into the linear transverse momentum of singlephotons, and use the 1024-dimensional QRAC GDW to certify that it is an irreducible quantum system. To achieve this, first, we show that the ASP [Eq. (1)] can be written as a simple function of the detection events. Then, we observe that our recorded statistics violate the second highest ASP bound, $Q_{512} Q_{2}$, given in Table I, thus, ensuring that it is an irreducible 1024-dimensional quantum system.

In the 1024-dimensional QRAC GDW, Bob measures the elements of the two 1024-dimensional MUBs given in
TABLE I. Relevant cases for a 1024-dimensional system and the respective optimal ASPs [Eq. (1)] considering each product structure. The full table can be found in the Supplemental Material [39].

\begin{tabular}{ll}
\hline \hline Case & Optimal $\bar{p}$ \\
\hline$Q_{1024}$ & 0.515625 \\
$Q_{512} Q_{2}$ & 0.500980 \\
$Q_{512} C_{2}$ & 0.500973 \\
$Q_{32} Q_{32}$ & 0.500521 \\
$\left(Q_{2}\right)^{10}$ & 0.500493 \\
$Q_{2} C_{512}$ & 0.500489 \\
$C_{1024}$ & 0.500488 \\
\hline \hline
\end{tabular}

the Supplemental Material [39]. We denote the MUB states by $\left|m_{j}^{y}\right\rangle$, where $y=1,2$ defines the measuring base $\mathrm{MUB}_{1}$ or base $\mathrm{MUB}_{2}$, and $j=1, \ldots, 1024$ denotes the state of a given base. Alice's state is written in terms of the two input dits $x_{1}$ and $x_{2}$ as an equal superposition of the states Bob would need to guess $x_{y}$ correctly

$$
\left|\Psi_{x_{1} x_{2}}\right\rangle=\frac{1}{N}\left(\left|m_{x_{1}}^{1}\right\rangle+\operatorname{sgn}\left(\left\langle m_{x_{1}}^{1} \mid m_{x_{2}}^{2}\right\rangle\right)\left|m_{x_{2}}^{2}\right\rangle\right),
$$

where $N=\sqrt{2\left(1+\frac{1}{32}\right)}$ is a normalization factor and sign is the sign function. The optimality of the encoded states (2), and the use of MUBs is derived in the Supplemental Material [39].

For the experimental test, we resort to the setup depicted in Fig. 2. At the state preparation block, the single-photon regime is achieved by heavily attenuating optical pulses with well calibrated attenuators. An acousto-optical modulator (AOM) placed at the output of a continuous-wave laser operating at $690 \mathrm{~nm}$ is used to generate the optical pulses. The average number of photons per pulse is set to $\mu=0.4$. In this case, the probability of having non-null pulses is $P(n \geq 1 \mid \mu=0.4)=33 \%$. Pulses containing only one photon are the majority of the non-null pulses generated and accounts to $82 \%$ of the experimental runs. Thus, our source is a good approximation to a nondeterministic single-photon source, which is commonly adopted in quantum communications [2].

The single-photons are then sent through two spatial light modulators, SLM1 and SLM2, addressing an array of $32 \times 32$ transmissive squares. The square side is $a=$ $96 \mu \mathrm{m}$ and they are equally separated by $\delta=160 \mu \mathrm{m}$ [see Fig. 2(b)], thus, effectively creating a 1024-dimensional quantum state defined in terms of the number of modes available for the photon transmission over the SLMs [5,21$23,33,34]$. Specifically, the state of the transmitted photon is given by $|\Psi\rangle=(1 / \sqrt{C}) \sum_{l=-l_{N_{C}}}^{l_{N_{c}}} \sum_{v=-l_{N_{r}}}^{l_{N_{r}}} \sqrt{t_{l v}} e^{-i \phi_{l v}}\left|c_{l v}\right\rangle$, where $\left|c_{l v}\right\rangle$ is the logical state representing the photon transmitted by the $(l, v)$ square. $t_{l v}$ represents the 
(a)

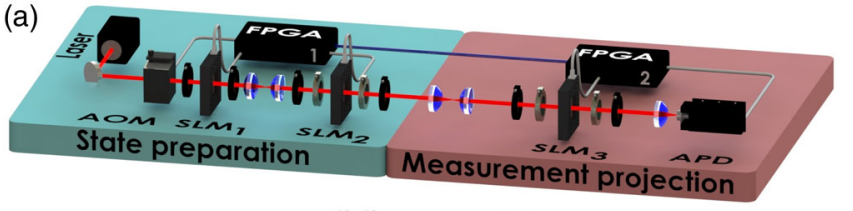

(b)

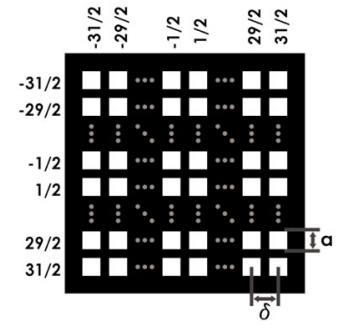

FIG. 2. (a) Experimental setup. We employ a prepareand-measure scheme to generate and project spatial qudits, encoded into the linear transverse momentum of single-photons. At the state preparation block, the spatial encoding is applied through two spatial light modulators (SLMs), and the state projection is likewise performed by a SLM combined with a pointlike avalanche single-photon detector (APD) at the measurement projection block (see main text for details). (b) The $32 \times 32$-square mask addressed by the SLMs.

transmission and $\phi_{l v}$ the phase-shift given by the $(l, v)$ square. The transmission of each square is controlled by the SLM1, which is configured for amplitude-only modulation. The phases $\phi_{l v}$ are controlled by SLM2 working on the configuration of phase-only modulation [22]. $N_{c}$ and $N_{r}$ represent the number of columns and rows, respectively. For simplicity, we define $l_{N_{c}} \equiv\left(N_{c}-1\right) / 2, l_{N_{r}} \equiv\left(N_{r}-1\right) / 2$, and $C$ is the normalization factor.

At the measurement block, we use a similar scheme to the one used in the state preparation block. It consists of a SLM3, also configured for phase-modulation, and a "pointlike" avalanche single-photon detector (APD). As explained in detail in $[5,22]$, by placing the pointlike APD at the SLM3 far-field (FF) plane, and properly adjusting the $(l, v)$ square phase shifts, Bob can detect any state $\left|m_{j}^{y}\right\rangle$ required for the 1024-dimensional QRAC session. The pointlike APD is composed of a pinhole (aperture of $10 \mu \mathrm{m}$ diameter) fixed at the center of the FF plane, followed by the APD module. In this case, the probability of photon detection is proportional to the overlap between the prepared and detected states. For the case of a $d$-dimensional QRACs implemented with a single-detector scheme, we show in the Supplemental Material (see [39] and Refs. $[4,5,9,13]$ therein) that the ASP function can be written as

$$
\bar{p}=\frac{D_{1}}{D_{1}+D_{2}} .
$$

First, we consider the events with $x_{y}=j$ (again, $j=$ $1, \ldots, 1024$ denotes the state of a given base) and define the total number of such events to be $X_{1}$. Then, we define
$D_{1}$ as the number of "clicks" recorded in the experiment in those cases. Likewise, we denote $X_{2}$ to be the number of events where $x_{y} \neq j$ and define $D_{2}$ to be the clicks in those cases.

By means of two field-programmable gate array (FPGA) electronic modules, we are able to automate and actively control both blocks of the setup. At the state preparation block, since the state $|\Psi\rangle$ needs to be randomly selected from the set of states defined by the 1024-dimensional QRACs, a random number generator (QRNG-Quantis) is connected to FPGA1. FPGA1 controls the optical pulse production rate by the $\mathrm{AOM}$, set at $60 \mathrm{~Hz}$ as limited by the refresh rate of the SLMs. Each attenuated optical pulse corresponds to an experimental round. At the measurement block, a second QRNG is connected to FPGA2, providing an independent and random selection for the projection $\left|m_{j}^{y}\right\rangle$ at each round. FPGA2 also records whether a detection event occurs. The overall detection efficiency is $13 \%$. The protocol is executed as follows: In each round, FPGA1 reads the dits $x_{1}$ and $x_{2}$ produced by its QRNG. Then, FPGA1 calculates the amplitude and phase of each $(l, v)$ square of SLM1 and SLM2 to encode the state $\left|\Psi_{x_{1} x_{2}}\right\rangle$ onto the spatial profile of the single-photon in that experimental round. Simultaneously, FPGA2, reads from its QRNG the value of $y$ and $j$. Similar to what is done in the state preparation block, FPGA2 also calculates the phase for each $(l, v)$ square in SLM3 to implement the chosen projection $\left|m_{j}^{y}\right\rangle$. The amplitude and relative phase for each SLM was previously characterized in order to obtain the modulation curves as a function of its grey level. In this experiment, this is necessary to dynamically generate all possible states, as it would be unfeasible to prerecord predefined masks for the SLMs on the FPGAs for each one of the $1024^{2}$ required initial states.

The experiment continuously ran over 316 hours. In this way, the statistics fluctuations observed for $D_{1}$ and $D_{2}$ were sufficiently small to unambiguously certify the generation of an irreducible 1024-dimensional quantum system. The

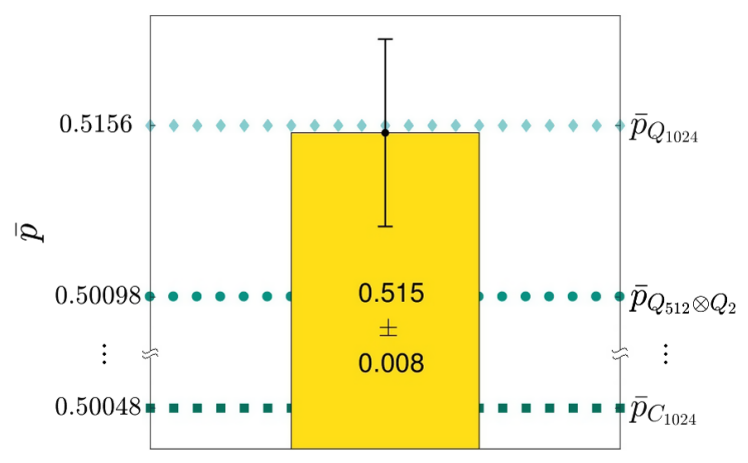

FIG. 3. Experimental results. We experimentally observe $\bar{p}=0.515 \pm 0.008$, violating the second highest ASP bound $\bar{p}_{Q_{512} \otimes Q_{2}}$ (see Table I). The error bar is calculated assuming Poissonian statistics for a photon detection event. 
overall visibility in our system is $97.00 \pm 0.07 \%$ and the corresponding recorded average success probability is $\bar{p}=0.515 \pm 0.008$. In Fig. 3, we compare it with the second highest ASP bound shown in Table I, associated with a composite system of the type $Q_{512} Q_{2}$. This certifies, only from the statistics recorded, that the generated state is not encoded using noncoupled different degrees of freedom of a photon, for instance polarization and momentum, thus, ensuring it to be an irreducible 1024-dimensional quantum system that can provide all the advantages known for highdimensional quantum information processing, in the sense explained in [31].

Conclusion.-Dimension witnesses are practical protocols on the field of quantum information as they allow one to obtain information regarding unknown quantum states $[25,26]$. They are especially appealing while addressing the generation and characterization of high-dimensional quantum states, where quantum tomography demands at least $d^{2}$ measurements [24]. In general, DWs are functions of only a few measurement outcome probabilities and allow for assessments on the dimension required to describe a given quantum state in a device-independent way [4,25-30]. Here, we give a step further by introducing a new class of DW, which certifies the dimension of the system, and has the new distinct feature of allowing the identification of whether a high-dimensional system is irreducible. The application of this new feature is of broad relevance for several new architectures aiming for high-dimensional quantum information processing [4-16], and the understanding of macroscopic quantumness [3]. We demonstrate the practicability of our technique by using it to certify the generation of an irreducible 1024-dimensional photonic quantum state encoded into the linear transverse momentum of singlephotons transmitted by programable diffractive apertures which have been used for several high-dimensional quantum information processing tasks [5,35,45-47].

This work was supported by First TEAM Grant No. 20161/5, NCN Sonata Grant No. UMO-2014/14/E/ST2/00020, Fondecyt Grants No. 1160400, No. 11150324, No. 1150101, and the Millennium Institute for Research in Optics, MIRO. E. A. A. acknowledges support from CONACyT. J. F. B. acknowledges support from Fondecyt 3170307. J. C. acknowledges support from Fondecyt 3170596. D. M. and M. A. acknowledge support from CONICYT.

E. A. A. and M. F. contributed equally to this work.

[1] T. D. Ladd, F. Jelezko, R. Laflamme, Y. Nakamura, C. Monroe, and J. L. O'Brien, Nature (London) 464, 45 (2010).

[2] Z.-S. Yuan, X.-H. Bao, C.-Y. Lu, J. Zhang, C.-Z. Peng, and J.-W. Pan, Phys. Rep. 497, 1 (2010).

[3] N. Gisin and F. Fröwis, arXiv:1802.00736.
[4] V. D’Ambrosio, F. Bisesto, F. Sciarrino, J. F. Barra, G. Lima, and A. Cabello, Phys. Rev. Lett. 112, 140503 (2014).

[5] S. Etcheverry, G. Cañas, E. S. Gómez, W. A. T. Nogueira, C. Saavedra, G. B. Xavier, and G. Lima, Sci. Rep. 3, 2316 (2013).

[6] W.-B. Gao, C.-Y. Lu, X.-C. Yao, P. Xu, O. Guhne, A. Goebel, Y.-A. Chen, C.-Z. Peng, Z.-B. Chen, and J.-W. Pan, Nat. Phys. 6, 331 (2010).

[7] A. C. Dada, J. Leach, G. S. Buller, M. J. Padgett, and E. Andersson, Nat. Phys. 7, 677 (2011).

[8] J. T. Barreiro, N. K. Langford, N. A. Peters, and P. G. Kwiat, Phys. Rev. Lett. 95, 260501 (2005).

[9] R. Fickler, R. Lapkiewicz, W. N. Plick, M. Krenn, C. Schaeff, S. Ramelow, and A. Zeilinger, Science 338, 640 (2012).

[10] M. Krenn, M. Huber, R. Fickler, R. Lapkiewicz, S. Ramelow, and A. Zeilinger, Proc. Natl. Acad. Sci. U.S.A. 111, 6243 (2014).

[11] X.-C. Yao, T.-X. Wang, P. Xu, H. Lu, G.-S. Pan, X.-H. Bao, C.-Z. Peng, C.-Y. Lu, Y.-A. Chen, and J.-W. Pan, Nat. Photonics 6, 225 (2012).

[12] T. Monz, P. Schindler, J. T. Barreiro, M. Chwalla, D. Nigg, W. A. Coish, M. Harlander, W. Hänsel, M. Hennrich, and R. Blatt, Phys. Rev. Lett. 106, 130506 (2011).

[13] X.-L. Wang, L.-K. Chen, W. Li, H.-L. Huang, C. Liu, C. Chen, Y.-H. Luo, Z.-E. Su, D. Wu, Z.-D. Li, H. Lu, Y. Hu, X. Jiang, C.-Z. Peng, L. Li, N.-L. Liu, Y.-A. Chen, C.-Y. Lu, and J.-W. Pan, Phys. Rev. Lett. 117, 210502 (2016).

[14] R. Barends et al., Nature (London) 508, 500 (2014).

[15] M. Malik, M. Erhard, M. Huber, M. Krenn, R. Fickler, and A. Zeilinger, Nat. Photonics 10, 248 (2016).

[16] R. Fickler, G. Campbell, B. Buchler, P. K. Lam, and A. Zeilinger, Proc. Natl. Acad. Sci. U.S.A. 113, 13642 (2016).

[17] G. M. D'Ariano and P. Lo Presti, Phys. Rev. Lett. 86, 4195 (2001).

[18] G. M. D'Ariano, L. Maccone, and P. Lo Presti, Phys. Rev. Lett. 93, 250407 (2004).

[19] D. F. V. James, P. G. Kwiat, W. J. Munro, and A. G. White, Phys. Rev. A 64, 052312 (2001).

[20] R. T. Thew, K. Nemoto, A. G. White, and W. J. Munro, Phys. Rev. A 66, 012303 (2002).

[21] G. Lima, E. S. Gómez, A. Vargas, R. O. Vianna, and C. Saavedra, Phys. Rev. A 82, 012302 (2010).

[22] G. Lima, L. Neves, R. Guzmán, E. S. Gómez, W. A. T. Nogueira, A. Delgado, A. Vargas, and C. Saavedra, Opt. Express 19, 3542 (2011).

[23] D. Goyeneche, G. Cañas, S. Etcheverry, E. S. Gómez, G. B. Xavier, G. Lima, and A. Delgado, Phys. Rev. Lett. 115, 090401 (2015).

[24] W. K. Wootters and B. D. Fields, Ann. Phys. (N.Y.) 191, 363 (1989).

[25] N. Brunner, S. Pironio, A. Acin, N. Gisin, A. A. Méthot, and V. Scarani, Phys. Rev. Lett. 100, 210503 (2008).

[26] R. Gallego, N. Brunner, C. Hadley, and A. Acín, Phys. Rev. Lett. 105, 230501 (2010).

[27] J. Ahrens, P. Badziag, A. Cabello, and M. Bourennane, Nat. Phys. 8, 592 (2012).

[28] M. Hendrych, R. Gallego, M. Micuda, N. Brunner, A. Acin, and J. P. Torres, Nat. Phys. 8, 588 (2012).

[29] N. Brunner, M. Navascués, and T. Vértesi, Phys. Rev. Lett. 110, 150501 (2013). 
[30] J. Bowles, M. T. Quintino, and N. Brunner, Phys. Rev. Lett. 112, 140407 (2014).

[31] W. Cong, Y. Cai, J.-D. Bancal, and V. Scarani, Phys. Rev. Lett. 119, 080401 (2017).

[32] A. Ambainis, A. Nayak, A. Ta-Shma, and U. Vazirani, in Proceedings of the Thirty-first Annual ACM Symposium on Theory of Computing, STOC '99 (ACM, New York, 1999) pp. 376-383.

[33] L. Neves, G. Lima, J. G. Aguirre Gómez, C. H. Monken, C. Saavedra, and S. Pádua, Phys. Rev. Lett. 94, 100501 (2005).

[34] G. Lima, A. Vargas, L. Neves, R. Guzmán, and C. Saavedra, Opt. Express 17, 10688 (2009).

[35] G. Cañas, N. Vera, J. Cariñe, P. González, J. Cardenas, P. W. R. Connolly, A. Przysiezna, E. S. Gómez, M. Figueroa, G. Vallone, P. Villoresi, T. F. da Silva, G. B. Xavier, and G. Lima, Phys. Rev. A 96, 022317 (2017).

[36] M. Czechlewski, D. Saha, and M. Pawłowski, arXiv:1803 .05245 .

[37] E. Aguilar, J. Borkała, P. Mironowicz, and M. Pawłowski, arXiv:1709.04898.

[38] M. Farkas, arXiv:1803.00363.

[39] See Supplemental Material at http://link.aps.org/ supplemental/10.1103/PhysRevLett.120.230503, which includes Refs. [40-42] for previous results on QRACs and majorization.
[40] A. Ambainis, D. Leung, L. Mancinska, and M. Ozols, Quantum Random Access Codes with Shared Randomness, Master's thesis, University of Waterloo (2009).

[41] A. Tavakoli, A. Hameedi, B. Marques, and M. Bourennane, Phys. Rev. Lett. 114, 170502 (2015).

[42] A. Marshall, I. Olkin, and B. Arnold, Inequalities: Theory of Majorization and Its Applications, Springer Series in Statistics (Springer, New York, 2010).

[43] We also note that numerical evidence on small dimensional examples suggests that, if the quantum states are in a product form, then entangling measurements do not improve the ASP.

[44] W. H. Press, S. A. Teukolski, W. T. Vetterling, and B. P. Flannery, Numerical Recipes (Cambridge University Press, Cambridge, 1992).

[45] B. Marques, A. A. Matoso, W. M. Pimenta, A. J. GutiérrezEsparza, M. F. Santos, and S. Pádua, Sci. Rep. 5, 16049 (2015).

[46] G. Cañas, S. Etcheverry, E. S. Gómez, C. Saavedra, G. B. Xavier, G. Lima, and A. Cabello, Phys. Rev. A 90, 012119 (2014).

[47] M. A. Solís-Prosser, M. F. Fernandes, O. Jiménez, A. Delgado, and L. Neves, Phys. Rev. Lett. 118, 100501 (2017). 